\title{
HUMAN FACTORS AND HIGHWAY-ACCIDENT CAUSATION: SOME THEORETICAL CONSIDERATIONS
}

\author{
Michael Sivak \\ Ilighway Safety Research Institute, University of Michigan, Ann Arbor, MI 48109, U.S.A.
}

(Received 12 February 1980; in revised form 30 April 1980)

\begin{abstract}
Traditional correlational analyses of human skills and highway accidents have not been very productive. Some of the likely reasons for this are discussed. An alternative approach to accident-causation research is outlined in which the importance of a skill with good face validity to driving is assessed in terms of its sensitivity to frequently occurring transient human states such as fatigue, stress and alcohol intoxication.
\end{abstract}

It is almost an axiom in highway safety research that traffic accidents are caused primarily by human errors, as opposed to vehicular malfunctions or adverse environmental conditions. Indeed, a recent in-depth accident investigation [Treat et al., 1977] cited human factors as definite or probable causes in $93 \%$ of the 420 accidents under investigation. Thus, it is not surprising that a substantial amount of research has been devoted to the investigation of the correlations between accidents and performance of a variety of skills, in efforts to identify skills critical to driving. However, results of such studies (e.g. the visual-skills study of Henderson and Burg [1974] and a variety of studies reviewed by Goldstein [1964]) generally yield disappointingly low correlations. Such findings indicate that the performance of each of a variety of skills can account for only a fraction of the variance exhibited in the accident record.

This paper presents arguments that: (1) indicate the likely reasons why none of the logically important skills have been shown to account for a substantial portion of the variance of the accident data, and (2) suggest an alternative approach in accident-causation research.

There are two principal problems in the approach of correlating accident records with human performance measures. First, as Goldstein has pointed out, "... accident involvement is not a highly stable characteristic of individual drivers [Goldstein, 1963, p. 2]," since there is only a low correlation between accidents in one period and accidents in another period. Therefore, "... accident involvement of individuals is not highly predictable from measurements of [relatively] stable characteristics [Goldstein, 1963, p. 2]," such as, for example, visual skills.

Second, current correlational studies attempt to relate events occurring in two different settings: The accidents occur in the "real-life" domain, while measures of human performance are usually obtained in the laboratory. The two settings probably differ considerably in the sets of transient human states present (e.g. fatigue, stress, alcohol intoxication). Moreover, it is well known that such transient human states affect a range of human skills, including some with good face validity to driving. For example, fatigue from prolonged (actual and simulated) driving has been shown to affect perception[Brown, 1967], decision-making [Brown. Tickner and Simmonds, 1970], and psychomotor performance [Dureman and Boden. 1972]. Therefore, it is not known how well, for example, laboratory-measured dynamic visual acuity replicates actual "on-the-road" dynamic visual acuity, since the "on-the-road" acuity may differ considerably from that measured in the laboratory.

These arguments suggest that even if, for example, the actual "on-the-road" dynamic visual acuity were critical to safe driving, correlational analyses of laboratory-measured dynamic visual acuity and accident involvement would not necessarily yield high correlations. Thus, not only have past studies not found high correlations, but future studies are also unlikely to do so: These studies are unlikely to identify the critical skills, which when deficient, cause accidents.

What then are potentially more fruitful areas of accident-prevention research? The most direct implication of the foregoing is that any correlational study should obtain the performance measures in a real-life setting. Optimally, such measures would be gathered by stopping motorists and testing them at the side of the road by means of a portable tester. No claim is 
being made that the transient human states of a motorist undergoing such a test will be the same as before he or she was stopped. Nevertheless, they are likely to be more similar than transient states present in the laboratory. However, various considerations (ethical, legal and methodological) make this option not very feasible. Moreover, the problem of the instability of individual accident records over time would still be present.

An alternative approach would abandon the correlational analysis altogether. There are three major aspects of this approach:

(1) The causal chain is assumed to be as follows: Transient human states affect human skills which, in turn, affect driving (i.e. affect the probability of accidents and near accidents). This sequence is depicted schematically in Fig. 1.

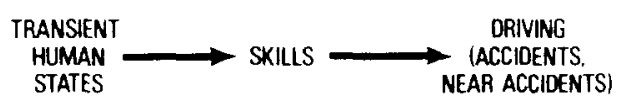

Fig. I. The assumed sequence in accident causation.

Relevant to this position. Shinar, McDonald and Treat [1978] identified critical interactions of human states and human behaviors in the causal chain of several of the accidents they investigated. (The sequence depicted in Fig. I does not apply to the relatively few accidents precipitated by a sudden vehicular malfunction, such as brake failure, or a sudden environmental factor, such as a muffler falling down from a vehicle immediately ahead.)

(2) The link between a variety of skills (with good face validity to driving) and driving performance is assumed. Because of the methodological problems outlined above, the actual relation cannot be experimentally assessed.

(3) The emphasis is placed on the effects of transient human states on skills: The critical skills for driving are assumed to be those face-valid skills that are most affected by changes in frequently occurring transient human states.

The underlying logic is that we cannot ignore the presence of various sets of transient states: People have to drive to work under the influence of family and/or employment stress; people have to drive home fatigued after a full day of work; people do (although they generally do not have to) drive while intoxicated. Therefore, if Skill A is relatively insensitive to a variety of frequently occurring transient human states, while Skill B is highly influenced by them (where both $\mathrm{A}$ and $\mathrm{B}$ are a priori important in terms of their face validity to driving), then Skill $B$ is more critical to safe driving than Skill $A$. This reasoning is less circular than it might seem at first glance: We have some good intuitions about what skills are used in driving, but we do not know their relative importance, and that is what correlational analyses have attempted to determine.

It can be argued that, in general, the relevant driving skills can be divided into four broad categories: attentional/scanning skills, perceptual/sensory skills, cognitive/decision/informationprocessing skills, and motor skills. Furthermore, it can be argued that the driving task is a series of simultaneous and continuous chains involving essentially serial and iterative utilization of various skills from these catcgories. Therefore, an interruption anywhere in any of the chains is potentially dangerous. The logic of the present approach suggests that as long as the chains are intact, one skill is not more important than other skills. What is crucial is that the serial processes of attention, information acquisition, decision-making, and motor execution repeat themselves continuously.

It is proposed that the driving task does not exceed human capabilities in any of these skill areas, given a sober, physically and mentally healthy individual who is not under the influence of fatigue, acute stress, or other undesirable transient states. (The few exceptions include situations involving sudden vehicular failures or sudden environmental aberrations.) This position is defensible on the grounds of the relatively high level of ergonomic considerations going into the design of present vehicles and highways. Furthermore, there is also some empirical evidence on this point. Brown et al.[1968], for example, have shown that $80 \%$ of the fatalities in their sample had had one stressful event in the 24-hour period preceding the accident. Similarly, McMurray's data[1970] indicate that people involved in divorce proceedings have double the accident rate of control motorists. 
The "ideal" (i.e. sober, healthy, not fatigued or stressed) driver might be, however, an exception and not the rule. Therefore, it is important to determine the effects of frequently occuring transient states on performance of skills with face validity to driving. In other words, future research should be directed towards obtaining information to fill cells of a matrix such as the one depicted in Table 1.

Table 1. Matrix of needed information on the effects of transient human states on human skills with face validity to driving

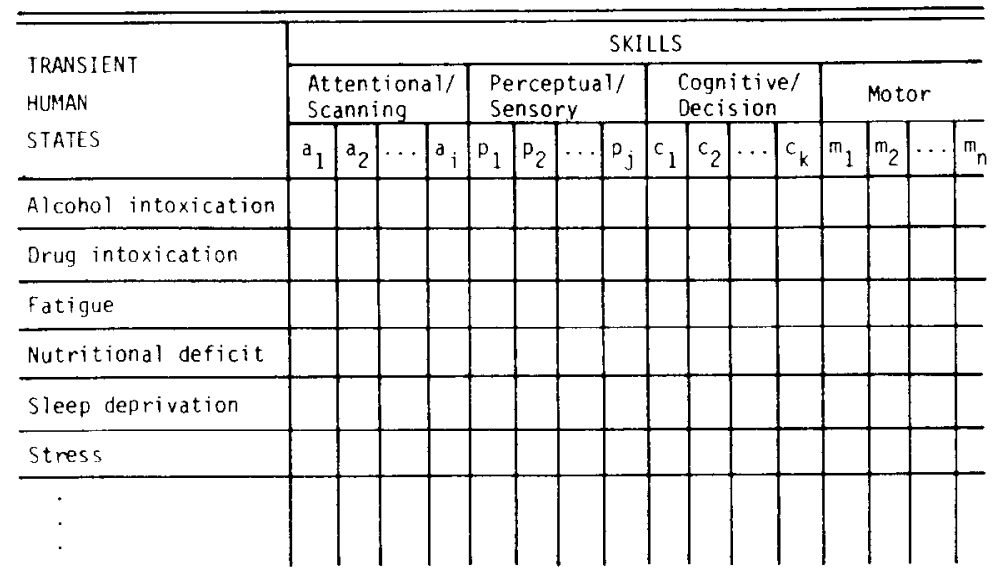

Some information can already be entered into several of the cells in Table 1 on the basis of past research findings. Examples of known relations include fatigue effects on visual contrast sensitivity [Hartman, 1963], risk-taking[Brown, Tickner and Simmond, 1970], and a range of motor skills [Welford, 1968]; alcohol effects on dynamic visual acuity [Brown et al., 1975], and glare sensitivity[Adams, Brown and Flom, 1976]; and stress effects on reaction time to lights [Weltman and Egstrom, 1971]. What is needed is rigorous research and analyses of the magnitude of the effects of transient states on a variety of skills with good face validity to driving.

A concrete example of the application of the proposed approach is given by analysis of the results of the following two studies dealing with static and dynamic visual acuity (skills with relatively good face validity to driving). Adams et al.[1975] have found that static visual acuity is unaffected by alcohol or marijuana intoxication. On the other hand, the results of Brown et al.[1975] indicate a significant effect of alcohol and marijuana on dynamic visual acuity. Thus, dynamic visual acuity has been shown to be more affected by frequently present transient human states (i.e. alcohol and marijuana intoxication) than static visual acuity. Therefore, according to the present rationale, dynamic visual acuity would be rated as more critical to safe driving than static visual acuity. (Obviously, before reaching any firm conclusions, effects of other transient states on both of the skills in question would have to be ascertained.)

There are three sources of difficultics in carrying out the rescarch called for by the present viewpoint that have to be recognized and resolved. First, there are obvious ethical barriers to creating undesirable, albeit transient, human states (e.g. stress), especially when using uninformed subjects. Experimental designs have to be developed that would yield valid information within the constraint of informed subjects. Second, there are potential disagreements in assigning a degree of face validity to a particular skill. However, while some skills might indeed fall into such a grey category (e.g. short-term memory), most of the skills are likely to be rather unanimously judged as either clearly relevant (e.g. speed of decision-making) or clearly irrelevant (e.g. gustatory skills). Third, there are difficulties in evaluating certain skills because they are rather poorly operationally defined. This is especially true of attentional skills. Basic research is required to develop the needed operational definitions.

The practical applications of the information gained from the proposed research are two-fold. First, the skills shown to be susceptible to transient states should be the targets in driver training and licensing to develop a high level of proficiency so that even after the transient decrements the level remains sufficiently high. Second. the motoring public should be 
informed about the likely decrements in driving-related skills under real-life conditions. This would be especially applicable to skills that are not amenable to training.

In summary, if a human skill with good face validity to driving is susceptible to frequently occurring transient states, then such a skill is important to safe driving. This viewpoint suggests a need for extensive and intensive research on the effects of transient states on skills with face validity to driving. Furthermore, the susceptible skills should be the targets in driver education and licensing, as well as in public information campaigns, so as to minimize the negative consequences of the transient human states.

Acknowledgements_Appreciation is extended to Lyle D. Filkins, Paul Green. James E. Haney, A. Regula Herzog, Richard J. Kaplan. John R. Treat, and two anonymous reviewers who, while not necessarily in agreement with the present viewpoint, offered constructive criticism of earlier drafts of this paper.

\section{REFERENCES}

Adams A. J., Brown B. Flom M. C., Jones R. T. and Jampolsky A., Alcohol and marijuarla effects on static visual acuity. Am. J. Optom. Physiol. Opt. 52, 729-735, 1975.

Adams A. J., Brown B. and Flom M. C. Alcohol-induced changes in contrast sensitivity following high-intensity light exposure. Percept. Psychophys. 19, 219-225, 1976.

Brown B., Adams A. J., Haegerstrom-Portnoy G., Jones R. T. and Flom M. C., Effects of alcohol and marijuana on dynamic visual acuity: I. Threshold measurements. Percep. Psychophys. 18, 441-446, 1975.

Brown I. D., Decrement in skill observed after seven hours of car driving. Psychonom. Sci. 7, 131-132. 1967.

Brown I. D., Tickner A. H. and Simmonds D. C. V., Effects of prolonged driving on overtaking criteria. Ergonomics 13. 239-242, 1970.

Brown S. L., Bohnert P. J., Finch, J. R., Pokorny A. D. and Smith J. P., Alcohol Safety Study: Dricers Who Die. Baylor University College of Medicine, Houston, Texas. 1968.

Dureman E. I. and Boden C., Fatigue in simulated car driving. Ergonomics 15, 299-308, 1972

Goldstein L. G., Whither accident research? Traff. Saf. Res. Ret. 7(1). 2-4, 1963.

Goldstein L. G. Human variahles in traffic accidents A digest of research Traff Saf. Res Ren 8(1), 26-31, 1964.

Hartman E., Disability glare and discomfort glare. In Lighting Problems in Highway Traffic (Edited by E. Ingelstam). MacMillan, New York, 1963.

Henderson R. L. and Burg A., Vision and Audition in Driting. System Development Corporation. Santa Monica, California, November 1974.

McMurray L., Emotional stress and driving performance: The effects of divorce. Behav. Res. Highw. Saf. 1, 100-114, 1970.

Shinar D., McDonald S. T. and Treat J. R., The interaction between driver mental and physical conditions and errors causing traffic accidents: An analytical approach. J. Saf. Res. 10, 16-23, 1978.

Treat J. R.. Tumbas N. S. McDonald S. T., Shinar D.. Hume R. D.. Mayer R. E., Stansifer R. L. and Castellan N. J., Tri-level study of the causes of traffic accidents: Final report. Vol. I: Causal factor tabulations and assessments. Institute for Research in Public Safety, Indiana University, Bloomington, Indiana, March 1977

Welford A. T., Fundamentals of skill. Methuen, London. 1968.

Weltman G. and Egstrom G. H. Perceptual narrowing in novice divers. Hum. Factors 8, 499-506, 1966. 